\title{
Automated detection of COVID-19 using ensemble of transfer learning with deep convolutional neural network based on CT scans
}

\author{
Parisa gifani $^{1} \cdot$ Ahmad Shalbaf $^{2}\left(\right.$ Majid Vafaeezadeh $^{3}$
}

Received: 23 May 2020 / Accepted: 23 October 2020 / Published online: 16 November 2020

(c) CARS 2020

\begin{abstract}
Purpose COVID-19 has infected millions of people worldwide. One of the most important hurdles in controlling the spread of this disease is the inefficiency and lack of medical tests. Computed tomography (CT) scans are promising in providing accurate and fast detection of COVID-19. However, determining COVID-19 requires highly trained radiologists and suffers from inter-observer variability. To remedy these limitations, this paper introduces an automatic methodology based on an ensemble of deep transfer learning for the detection of COVID-19.

Methods A total of 15 pre-trained convolutional neural networks (CNNs) architectures: EfficientNets(B0-B5), NasNetLarge, NasNetMobile, InceptionV3, ResNet-50, SeResnet 50, Xception, DenseNet121, ResNext50 and Inception_resnet_v2 are used and then fine-tuned on the target task. After that, we built an ensemble method based on majority voting of the best combination of deep transfer learning outputs to further improve the recognition performance. We have used a publicly available dataset of CT scans, which consists of 349 CT scans labeled as being positive for COVID-19 and 397 negative COVID-19 CT scans that are normal or contain other types of lung diseases.

Results The experimental results indicate that the majority voting of 5 deep transfer learning architecture with EfficientNetB0, EfficientNetB3, EfficientNetB5, Inception_resnet_v2, and Xception has the higher results than the individual transfer learning structure and among the other models based on precision (0.857), recall (0.854) and accuracy (0.85) metrics in diagnosing COVID-19 from CT scans.

Conclusion Our study based on an ensemble deep transfer learning system with different pre-trained CNNs architectures can work well on a publicly available dataset of CT images for the diagnosis of COVID-19 based on CT scans.
\end{abstract}

Keywords COVID-19 · CT · Transfer learning · Convolutional neural network · Ensemble model

\section{Introduction}

COVID-19 is an infectious disease that has infected more than 4.5 million individuals all over the world until May 14 in 2020 [1]. The current tests for diagnosis of this disease are mostly based on reverse transcription-polymerase chain reaction (RT-PCR). However, RT-PCR test kits are in

Ahmad Shalbaf

shalbaf@sbmu.ac.ir

1 Department of Biomedical Engineering, Science and Research Branch, Islamic Azad University, Tehran, Iran

2 Department of Biomedical Engineering and Medical Physics, School of Medicine, Shahid Beheshti University of Medical Sciences, Tehran, Iran

3 School of Electrical Engineering, Iran University of Science and Technology, Tehran, Iran huge shortage and take $4-6 \mathrm{~h}$ to obtain results, which are a long time compared with the rapid spreading rate of COVID19. As a result, many infected cases cannot be detected promptly and continue to infect others unconsciously. So, many efforts have been done for alternative testing methods. Computed tomography (CT) scans devices are promising in serving as fast speed, more efficient and accessible testing manner [2]. The diagnosis of COVID-19 is based on the evaluation and assessment of CT images by the radiologist. But, this work is tedious and often has a high degree of inter-observer variability that results in uncertainty. Thus, to overcome the mentioned limitations, an automatic, reliable and reproducible approach using advanced machine learning is required. This system can overcome these limitations and can be utilized everywhere with no need for a highly trained radiologist. 
Different methods of machine learning are widely used in medical image processing. Among them, in recent years, deep learning methods have been introduced as the newest and most advanced methods. DL methods that rely on multi-layered neural networks can extract and learn increasingly complex, task-related features directly from the data. Recent developments in neural network architecture design and training have enabled researchers to solve previously intractable learning tasks of DL methods. But, these models need a lot of data for good training and prevent overfitting. To address this problem, transfer learning methods are introduced. Transfer learning is done by considering a standard neural architecture along with its pre-trained weights on large datasets and then fine-tuning the weights on the target task that has limited training data [3]. In other words, our strategy is to learn a powerful deep network to extract comprehensive visual features by pretraining on large datasets and then adapt the network weights of this pre-trained deep network to the target task with the small-sized dataset. As a result, several types of research in recent years have focused on the application of deep transfer learning in a wide range of medical image classification and recognition tasks with very success [4-7], especially in skin cancer [8], pneumonia detection [9], tumor classification [10], cardiovascular [11], ophthalmology [12], musculoskeletal [13] and histopathological images [14].

Medical images analysis from chest X-ray and CT images is very important for early and accurate diagnosis pneumonia and similar pulmonary disease and assists for effective treatments. Ke et al. [15] have proposed the hybrid neuro-heuristic and neuro-fuzzy methods for detection small changes in the structure of lung tissues and Chandra et al. [16] tuberculosisrelated abnormalities detection using hierarchical feature extraction method from the Chest X-ray images. Peláez et al. [17] have proposed a novel methodology for automated identification of interstitial lung abnormalities patterns using an ensemble of deep convolutional neural networks, and Gupta et al. [18] identify chronic obstructive pulmonary disease and fibrosis using extracting relevant features, feature selection and identification using a machine learning classifier in lung CT images. Recently, there have been some studies for automatic detection of COVID-19 based on CT scans using DL methods. Xu et al. have proposed multiple CNN models [19], Zheng et al. a 3D deep CNN (DeCoVNet) [20] and Wang et al. a modified inception transfer learning model [21] to detect COVID-19 based on chest CT scan. Also, Shan et al. employed the "VB-Net" neural network to segment COVID-19 infection regions [22] and Chen et al. UNet + + for identification of COVID-19 from CT scans [23]. The above studies suggest that DL is a feasible task, but were all evaluated in a limited setting.

The novelty and contribution of this paper are an automatic methodology based on an ensemble deep transfer learning system with different pre-trained CNNs architectures on a publicly available dataset of CT images for the diagnosis of COVID-19. We design the optimal combination of deep transfer learning outputs using an additional ensemble network.

\section{Material and method}

\section{Database}

We have used a publicly available COVID19-CT dataset [24], which consists of $349 \mathrm{CT}$ scans labeled as being positive for COVID-19 from 216 patient cases and 397 negative COVID19 CT scans that are normal or contain other types of lung diseases from 171 cases. The utility of this dataset has been confirmed by a senior radiologist in Tongji Hospital, Wuhan, China, who has performed diagnosis and treatment of a large number of COVID-19 patients between January and April. More details about the used dataset are described in this paper [20]. The minimum, maximum and average number of CT scans for a patient are 1.0, 16.0 and 1.6, respectively. These CT images have different sizes. The average, maximum and minimum height are 491, 1853 and 153 pixels, respectively. The average, maximum and minimum width are 383, 1485 and 124 pixels, respectively. Figure 1 shows some examples of the positive COVID-19 CT scans.

\section{Deep learning and Convolutional neural networks}

Deep learning algorithms compared with other conventional machine learning methods have become particularly popular for the diagnosis of diseases in medical imaging with considerable performance improvements. One of the most popular DL methods in the field of medical imaging is CNN [25]. It is the state-of-the-art DL methodology consisting of many stacked convolutional layers. The CNN structure comprises a convolutional layer, a maximum or average pooling layer, a nonlinear layer, batch normalization, fully connected (FC) layers and finally a softmax layer. Pooling layers are frequently used among convolutional layers to boost translational invariance and lessen feature map extent. Nonlinear layers (mostly ReLU function) are used to strengthen the network for solving nonlinear problems. Finally, FC layers prepare extracted features to be classified by the softmax layer.

\section{Transfer learning}

The numbers of parameters in the model increase as networks get deeper for improved learning efficiency. The deeper networks lead to the more complicated computations and the more demanding to training data. We have only 387 cases 
Fig. 1 Examples of positive COVID-19 CT scans

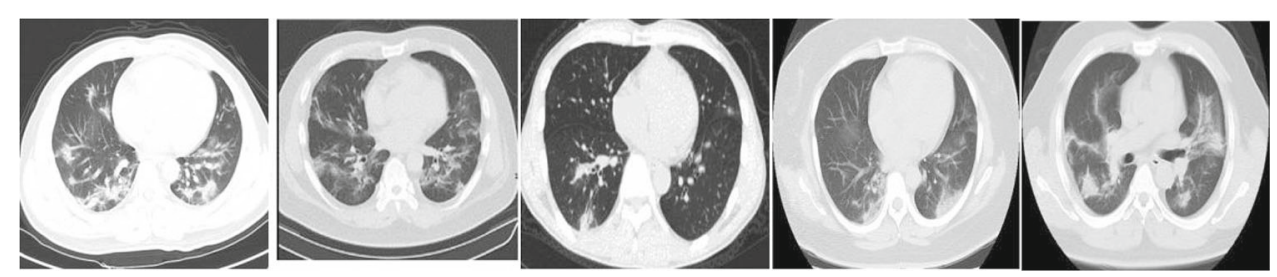

with 746 images to train, validate and test data. It seems too small to train a deep $\mathrm{CNN}$, and we need the transfer learning concept. Transfer learning employs take advantage of a pre-trained model (CNN model) on a huge database to help with the learning of the target task (e.g., diagnosing COVID-19 from CT scans) that has limited training data [26]. It means we transfer the information (learning expressive and generalizable feature representations) to our problem with an insufficient database. When applying this strategy, we have one problem. The large image data mostly belong to the general domain, such as cat, dog and chair, whereas our images are COVID-19 CTs with different visual appearances. As a result, the visual representations learned on these large images may not be able to represent CT images well. It makes the network extraction feature biased with the source data and less generalized on the target data. So, the pretrained models are then fine-tuned on our new dataset with a lesser number of training images. In other words, pre-trained CNN structures are modified to suit our task. This procedure is usually much faster than the conventional training of the CNN model with random weights. To train enormous parameters of a CNN model adequately, too much data are needed. We use transfer learning to compensate the lack of many datasets and achieving better outcomes. Several special CNN architectures are trained on very large amounts of images with many categories and then named pre-trained CNNs model. There are trained on ImageNet contains 14 million images of 1000 different categories from animals (dogs, cats, lions, ....) to objects (desks, pens, chairs, ...) [27]. EfficientNets(B0-B5) [28], NasNetLarge, NasNetMobile [29], InceptionV3 [30], ResNet-50 [31], SeResnet50 [32], Xception [33], DenseNet121 [34], ResNext50 [35] and Inception_resnet_v2 [36] are popular pre-trained CNNs. These networks have benefits for researchers such as lower training time, weaker and cheaper hardware requirements, and lower computational load.

\section{Training details}

We evaluated pre-trained CNNs by fine-tuning them on our clinical dataset, separately. For this reason, we adopted 15 pre-trained CNNs: EfficientNets(B0-B5), NasNetLarge, NasNetMobile, InceptionV3, ResNet-50, SeResnet50, Xcep- tion, DenseNet121, ResNext50 and Inception_resnet_v2. Image augmentation is used in our study. In addition to the initial dataset, another training dataset by the technique of data augmentation is generated. Data augmentation can be applied during training to reduce the overfitting problem of deep CNN. In this research, we applied randomly horizontally and vertically shift to an extent of $10 \%$ of the original dimensions. Also random rotation $\left(20^{\circ}\right)$ combined with small random zoom was applied to the training images. We also flip horizontally the images to increase the size of dataset. To fine-tuning all networks, we only used the convolutional part of each model's architecture, removing all fully connected layers. On top of the last convolutional layer, we added a global average pooling layer, followed by the final classification layer that uses softmax non-linearity. For fine-tuning the networks, all models were fine-tuned for 50 epochs using stochastic gradient descent (SGD) optimization with an initial learning rate of 0.0001 , Nesterov momentum 0.9 and the batch size of 32 . In all cases, the categorical cross-entropy was used as the loss function. Hyperparameters are tuned on the validation set. It should be noted that the input of each network is of a different size. So in the first step of data preparation, according to different sizes of model inputs, all images were resized to proper sizes and stored in separate folders. Table 1 compares the pre-trained CNN models. These models were trained using the same initialization and learning rate policies.

\section{Evaluation metrics}

Independently, 15 versions of the pre-trained CNNs model were fine-tuned and the prediction was used on the test set. Accordingly, the dataset was split into a training set, a validation set and a test set $(60 \%-15 \%-25 \%)$. Common classification metrics named: accuracy, precision, recall, F1 score and AUC, which is the area under the receiver operating characteristic (ROC) curve, were also used for evaluation of the proposed method. F1-score is the harmonic mean of precision and recall. AUC is a performance measurement for classification problems at various thresholds settings that represents a degree of separability and tells how much model is capable of distinguishing between classes. 
Table 1 Comparison of the analyzed pre-trained CNNs

\begin{tabular}{|c|c|c|c|c|c|c|}
\hline Architecture name & Year & Main contribution & Parameters & FLOP & \# trainable layers & Input shape \\
\hline EfficientNetB0 & 2019 & Compound scaling & $4,049,564$ & $0.39 \mathrm{~B}$ & 237 & $224 \times 224$ \\
\hline EfficientNetB1 & 2019 & Compound scaling & $6,575,232$ & $0.70 \mathrm{~B}$ & 338 & $240 \times 240$ \\
\hline EfficientNetB2 & 2019 & Compound scaling & $7,768,562$ & $1.0 \mathrm{~B}$ & 338 & $260 \times 260$ \\
\hline EfficientNetB3 & 2019 & Compound scaling & $10,783,528$ & $1.8 \mathrm{~B}$ & 383 & $300 \times 300$ \\
\hline EfficientNetB4 & 2019 & Compound scaling & $17,673,816$ & $4.2 \mathrm{~B}$ & 473 & $380 \times 380$ \\
\hline EfficientNetB5 & 2019 & Compound scaling & $28,513,520$ & $9.9 \mathrm{~B}$ & 575 & $456 \times 456$ \\
\hline NasnetLarge & 2017 & $\begin{array}{l}\text { Define blocks and cells in architectures } \\
\text { Find the best architectures by following a search strategy that } \\
\text { will maximize the performance }\end{array}$ & $84,916,818$ & $24 \mathrm{~B}$ & 1038 & $331 \times 331$ \\
\hline NasnetMobile & 2017 & $\begin{array}{l}\text { Define blocks and reduction cells in architectures } \\
\text { Find the best architectures by following a search strategy that } \\
\text { will maximize the performance }\end{array}$ & $4,269,716$ & $0.56 \mathrm{~B}$ & 768 & $224 \times 224$ \\
\hline Inception-V3 & 2015 & $\begin{array}{l}\text { Handles the problem of a representational bottleneck } \\
\text { Replace large size filters with small filters }\end{array}$ & $21,802,784$ & $5.7 \mathrm{~B}$ & 310 & $299 \times 299$ \\
\hline Inception-ResNet & 2016 & Uses split transform merge idea and residual links & $54,336,736$ & 13B & 779 & $299 \times 299$ \\
\hline ResNet 50 & 2016 & $\begin{array}{l}\text { Residual learning } \\
\text { Identity mapping based skip connections }\end{array}$ & $23,546,057$ & $4.1 \mathrm{~B}$ & 189 & $224 \times 224$ \\
\hline Xception & 2017 & Depth-wise convolution followed by point-wise convolution & $20,861,480$ & $8.4 \mathrm{~B}$ & 131 & $299 \times 299$ \\
\hline ResNeXt & 2017 & $\begin{array}{l}\text { Cardinality } \\
\text { Homogeneous topology } \\
\text { Grouped convolution }\end{array}$ & $23,048,137$ & $4 \mathrm{~B}$ & 215 & $224 \times 224$ \\
\hline SEResNet & 2017 & Models interdependencies between feature-maps & $26,092,144$ & $4 \mathrm{~B}$ & 286 & $224 \times 224$ \\
\hline DenseNet 121 & 2017 & Cross-layer information flow & $7,037,504$ & $3 \mathrm{~B}$ & 426 & $224 \times 224$ \\
\hline
\end{tabular}

\section{Results}

Independently, 15 versions of pre-trained CNNs model: EfficientNets(B0-B5), NasNetLarge, NasNetMobile, InceptionV3, ResNet-50, SeResnet50, Xception, DenseNet121, ResNext50 and Inception_resnet_v2 on ImageNet datasets were fine-tuned on our dataset of COVID19-CT images, with the goal of transferring the information into our task that has limited training data. DL was performed using Python version 3.5 programming language (Python Software Foundation, Beaverton, Oregon) with Keras version 2.1.5 software (GitHub, San Francisco, California) using a graphics processing unit (GeForce GTX 1080 Ti, NVIDIA, Santa Clara, California). Figures 2 and 3 show the value of the accuracy and loss function in the training sets for fine-tuning of different pre-trained CNN models, respectively. As shown in these figures, the model converges in the training process after the 50th epoch, and the data distribution ranges were narrow. So, after 50 steps of training, diagnostic accuracy was calculated using the test set. The results of transfer learning using different structures are illustrated in Table 2 by common classification metrics, accuracy, precision, recall, F1 score and AUC. The EfficientNetB0, EfficientNetB5 and InceptionV3 give higher accuracy (0.82) in the classification of CT images as compared to other architectures. But, the precision (0.847) and recall (0.822) metrics of the EfficientNetB0 model is the best among the other pre-trained CNNs model. Results of the ROC-AUC analysis used to assess the diagnostic ability and classification of CT images at different pre-trained CNN models are shown in Fig. 4. The DL architecture with the largest AUC was EfficientNetB0 (AUC: 0.907), InceptionV3 (AUC: 0.897) and EfficientNetB5 (AUC: 0.886).

To improve the classification accuracy, an ensemble method has been developed, where different architectures of deep transfer learning outputs as different classifiers are fused using additional work. A majority voting criterion was used by the ensemble of classifiers to assign the final prediction to the test data. For a given test image, the outputs of the classifiers are averaged to generate the final output of the ensemble. We experimented with ensembles of 3,5 , 7, 9, 11, 13 and 15 different architectures of deep transfer learning outputs. The experimental results indicate that the majority voting of 5 deep transfer learning architecture with EfficientNetB0, EfficientNetB3, EfficientNetB5, Inception_resnet_v2 and Xception achieves best results in diagnosing COVID-19 from CT scans. The performance of the ensemble model is given in Table 2. It was observed that the proposed approach using ensemble models using majority voting scheme for final prediction is better than individual models with higher accuracy $(0.85)$, precision $(0.857)$ and recall $(0.852)$. The confusion matrix for the best ensemble model is presented in Table 3. In this table, 22 images labeled 
Fig. 2 Training accuracy for different pre-trained $\mathrm{CNN}$ models

Fig. 3 Cross-entropy loss function for different pre-trained $\mathrm{CNN}$ models
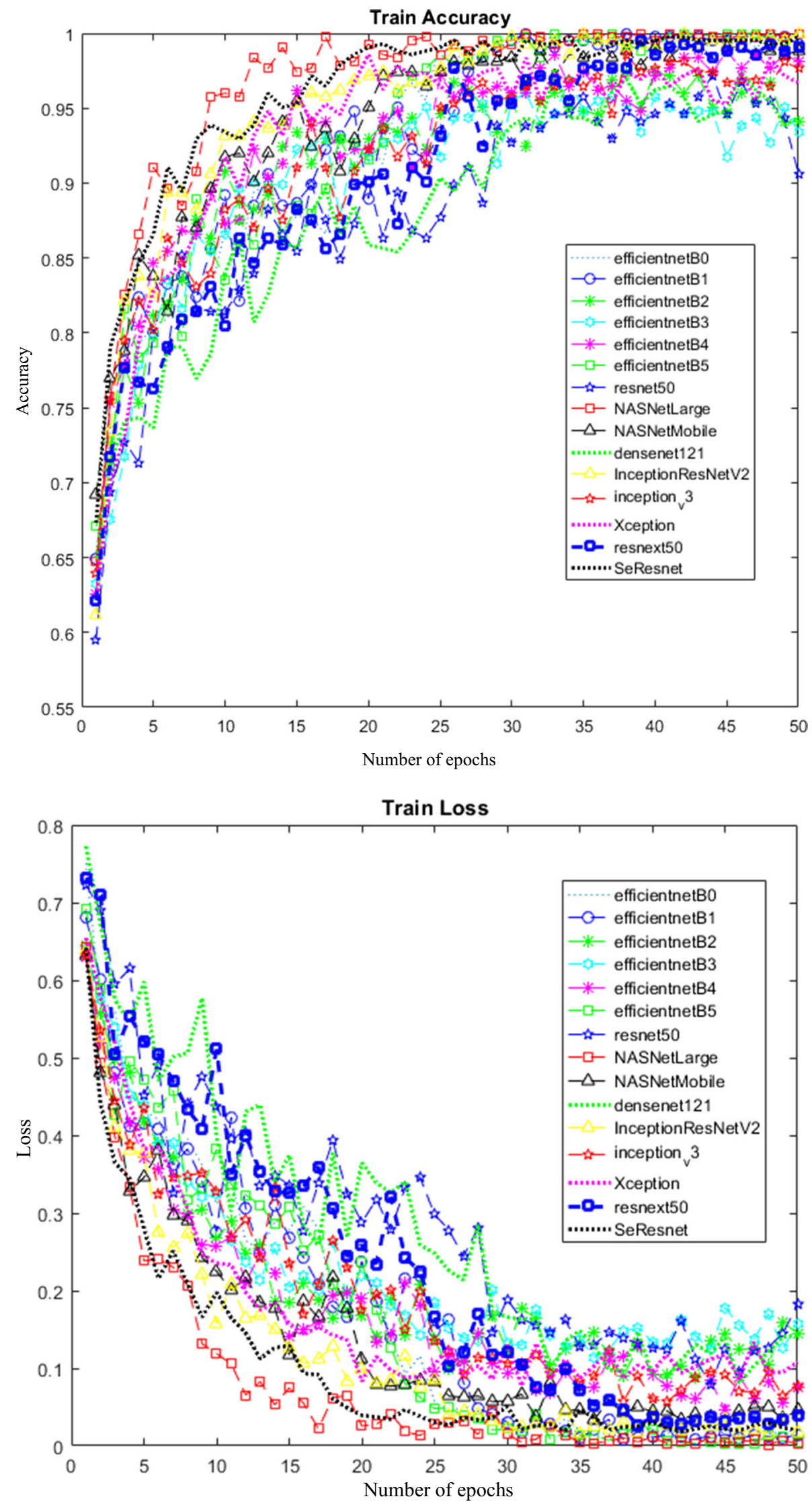
Table 2 Classification metrics on the test dataset using the different architecture of deep transfer learning models and also proposed ensemble method. For each model, average $( \pm$ std. performance measure is reported over the best 5 trained model checkpoints

\begin{tabular}{llllll}
\hline Model & Precision & Recall & F1-score & Accuracy & AUC \\
\hline EfficientNetB0 & $0.847( \pm 0.03)$ & $0.822( \pm 0.11)$ & $0.815( \pm 0.05)$ & $0.82( \pm 0.02)$ & $0.907( \pm 0.02)$ \\
EfficientNetB1 & $0.727( \pm 0.06)$ & $0.718( \pm 0.09)$ & $0.712( \pm 0.03)$ & $0.71( \pm 0.02)$ & $0.809( \pm 0.02)$ \\
EfficientNetB2 & $0.768( \pm 0.03)$ & $0.768( \pm 0.12)$ & $0.768( \pm 0.05)$ & $0.77( \pm 0.03)$ & $0.859( \pm 0.03)$ \\
EfficientNetB3 & $0.769( \pm 0.03)$ & $0.765( \pm 0.07)$ & $0.763( \pm 0.03)$ & $0.76( \pm 0.03)$ & $0.851( \pm 0.01)$ \\
EfficientNetB4 & $0.791( \pm 0.02)$ & $0.789( \pm 0.05)$ & $0.788( \pm 0.01)$ & $0.79( \pm 0.01)$ & $0.877( \pm 0.01)$ \\
EfficientNetB5 & $0.817( \pm 0.03)$ & $0.817( \pm 0.11)$ & $0.817( \pm 0.05)$ & $0.82( \pm 0.03)$ & $0.886( \pm 0.01)$ \\
Inception_resnet_v2 & $0.773( \pm 0.03)$ & $0.774( \pm 0.12)$ & $0.773( \pm 0.05)$ & $0.77( \pm 0.02)$ & $0.856( \pm 0.01)$ \\
InceptionV3 & $0.825( \pm 0.03)$ & $0.814( \pm 0.07)$ & $0.815( \pm 0.03)$ & $0.82( \pm 0.02)$ & $0.897( \pm 0.02)$ \\
NASNetLarge & $0.772( \pm 0.06)$ & $0.770( \pm 0.09)$ & $0.768( \pm 0.03)$ & $0.77( \pm 0.01)$ & $0.836( \pm 0.03)$ \\
NASNetMobile & $0.759( \pm 0.03)$ & $0.757( \pm 0.12)$ & $0.757( \pm 0.05)$ & $0.76( \pm 0.04)$ & $0.823( \pm 0.02)$ \\
ResNet50 & $0.807( \pm 0.03)$ & $0.808( \pm 0.11)$ & $0.807( \pm 0.05)$ & $0.81( \pm 0.03)$ & $0.875( \pm 0.01)$ \\
Xception & $0.738( \pm 0.06)$ & $0.739( \pm 0.09)$ & $0.738( \pm 0.03)$ & $0.74( \pm 0.04)$ & $0.782( \pm 0.04)$ \\
DenseNet121 & $0.768( \pm 0.03)$ & $0.768( \pm 0.03)$ & $0.768( \pm 0.03)$ & $0.77( \pm 0.02)$ & $0.868( \pm 0.04)$ \\
SeResnet50 & $0.755( \pm 0.03)$ & $0.745( \pm 0.07)$ & $0.745( \pm 0.03)$ & $0.75( \pm 0.02)$ & $0.818( \pm 0.02)$ \\
ResNext50 & $0.810( \pm 0.03)$ & $0.806( \pm 0.12)$ & $0.806( \pm 0.05)$ & $0.81( \pm 0.02)$ & $0.843( \pm 0.02)$ \\
Proposed ensemble & $0.857( \pm 0.02)$ & $0.854( \pm 0.05)$ & $0.852( \pm 0.01)$ & $0.852( \pm 0.01)$ & $0.91( \pm 0.01)$ \\
model & & & & & \\
\hline & & & & &
\end{tabular}

as negative COVID-19 are determined positive COVID-19 by our proposed method. The reason for this may be that using other types of lung diseases in test set is labeled as negative COVID-19.

\section{Discussion}

In this research, we investigate the performance of an ensemble deep learning structure for automated detection of COVID-19 on a publicly available dataset of CT images. We explored 15 state-of-the-art pre-trained CNN architectures which have trained and shown excellent performance on the ImageNet dataset, namely EfficientNets(B0B5), NasNetLarge, NasNetMobile, InceptionV3, ResNet50, SeResnet50, Xception, DenseNet121, ResNext50 and Inception_resnet_v2. Then, these pre-trained networks are fine-tuned on the target task that has limited training data. EfficientNetB0 model is the best among the other pre-trained CNNs model based on the accuracy (0.82), precision (0.847) and recall (0.822) metrics in the classification of CT images. Finally, to improve the classification accuracy, an ensemble method based on majority voting of different architectures of deep transfer learning outputs has been developed. We observe that the majority voting of prediction of 5 deep transfer learning architecture with EfficientNetB0, EfficientNetB3, EfficientNetB5, Inception_resnet_v2 and Xception is the best model with higher accuracy $(0.85)$, precision $(0.857)$ and recall (0.852) than the individual transfer learning models.
An ensemble of classifiers typically outperforms a single classifier due to reducing variance in the final prediction. In the context of deep transfer learning, an ensemble of classifiers can be built by different deep transfer learning by changing the architecture of the pre-trained CNN networks. Certain neural architectures facilitate transfer learning better than others for representation learning. A total of different deep transfer learning structures were used to form an ensemble of the classifier. We evaluate the efficacy of different deep transfer learning with different network architectures via extensive experiments. A total of 3, 5, 7, 9, 11, 13 and 15 different architectures of deep transfer learning were used to form an ensemble of classifier, and the final classification was done based on majority voting.

So, compared to other similar studies, this work has the advantage of comparing the deep transfer learning models with different pre-trained CNNs and an ensemble deep transfer learning system. The main drawback of the research can be considered the dataset size to train the networks. By performing regularization terms and simplifying deep models, we were able to overcome this problem. Our aim in the future is to further expand the experimental space by collecting more samples and employing the developed methodology on other CT images.

\section{Conclusion}

We perform a comprehensive study to systematically investigate the effects of different transfer learning structures with different pre-trained CNNs architectures and propose 

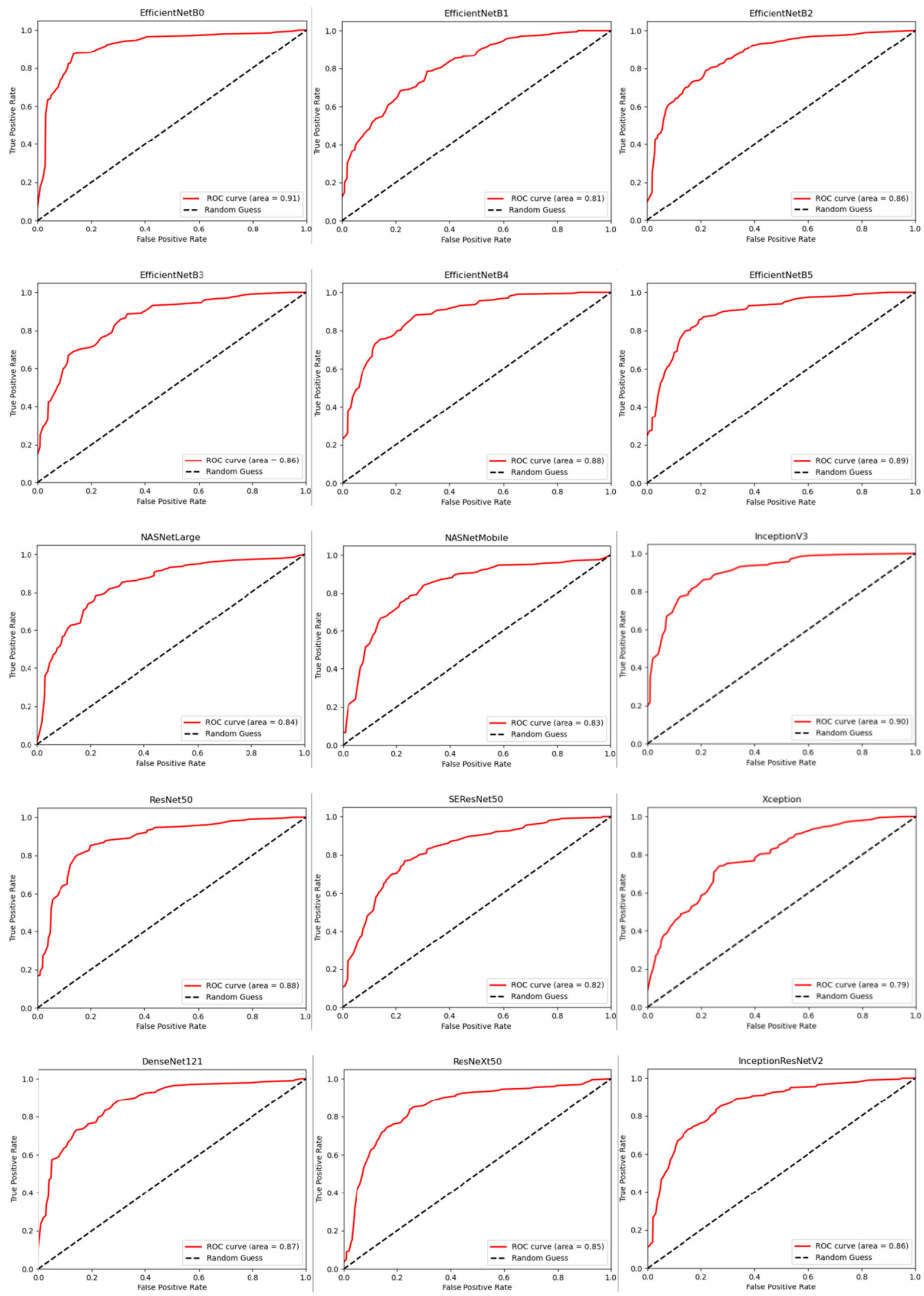

Fig. 4 ROC/AUC curves of different architecture of deep transfer learning models 
Table 3 Confusion matrix for the best ensemble model

\begin{tabular}{|c|c|c|c|}
\hline & & & \\
\hline & & COVID & Non-COVID \\
\hline 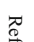 & COVID & 90 & 8 \\
\hline है & Non-COVID & 22 & 83 \\
\hline
\end{tabular}

a new ensemble approach, which synergistically integrates transfer learning strategies for COVID-19 diagnosis and provides insightful findings on a publicly available dataset of CT images. It was observed that the majority voting of 5 deep transfer learning architecture with EfficientNetB0, EfficientNetB3, EfficientNetB5, Inception_resnet_v2 and Xception has the higher results than the individual transfer learning structure based on precision (0.857), recall (0.854) and accuracy $(0.85)$ metrics. So, our proposed method can work well for the diagnosis of COVID-19 based on CT scans.

\section{Compliance with ethical standards}

Conflict of interest The authors declare that they have no conflict of interest.

Ethical approval All procedures performed in studies involving human participants were in accordance with the ethical standards of the institutional and/or national research committee and with the 1964 Helsinki Declaration and its later amendments or comparable ethical standards.

Informed consent Informed consent was obtained from all individual participants included in the study.

\section{References}

1. https://coronavirus.1point3acres.com/en/world

2. Bernheim A, Mei X, Huang M, Yang Y, Fayad ZA, Zhang N, Diao K, Lin B, Zhu X, Li K, Li S, Shan H, Jacobi A, Chung M (2020) Chest ct findings in coronavirus disease-19 (covid-19): relationship to duration of infection. Radiology 295(3):200463

3. Chuanqi T, Fuchun S, Tao K, Wenchang Z, Chao Y, Chunfang L (2018) A survey on deep transfer learning. In: The 27th international conference on artificial neural networks (ICANN 2018). Lecture Notes in Computer Science. Springer

4. Litjens G, Kooi T, Ehteshami Bejnordi B, Arindra Adiyoso Setio A, Ciompi F, Ghafoorian M, van der Laak JAWM, Ginneken B, Sánchez CI (2017) A survey on deep learning in medical image analysis. Med Image Anal 42:60-88

5. Janowczyk A, Madabhushi A (2016) Deep learning for digital pathology image analysis: a comprehensive tutorial with selected use cases. J Pathol Inform 7:29

6. Greenspan H, van Ginneken B, Summers RM (2016) Guest editorial deep learning in medical imaging: overview and future promise of an exciting new technique. IEEE Trans Med Imaging 35(5):1153-1159

7. Poplin R, Varadarajan AV, Blumer K, Liu Y, McConnell MV, Corrado GS, Peng L, Webster DR (2018) Prediction of cardiovascular risk factors from retinal fundus photographs via deep learning. Nat Biomed Eng 2:158-164

8. Esteva A, Kuprel B, Novoa RA, Ko J, Swetter SM, Blau HM, Thrun S (2017) Dermatologist-level classification of skin cancer with deep neural networks. Nature 542:115-118

9. Chouhan V, Singh SK, Khamparia A, Gupta D, Tiwari P, Moreira C, Dama`sevi`cius R, de Albuquerque VHC (2020) A novel transfer learning based approach for pneumonia detection in chest $\mathrm{x}$-ray images. Appl Sci 10(2):559

10. Huynh BQ, Li H, Giger ML (2016) Digital mammographic tumor classification using transfer learning from deep convolutional neural networks. J Med Imaging 3(3):034501

11. Litjens G, Ciompi F, Wolterink JM, de Vos BD, Leiner T, Teuwen J, Išgum I (2019) State-of-the-art deep learning in cardiovascular image analysis. JACC Cardiovasc Imaging 12(8):1549-1565

12. De Fauw J, Ledsam JR, Ronneberger O (2018) Clinically applicable deep learning for diagnosis and referral in retinal disease. Nat Med 24:1342-1350

13. Chea P, Mandell JC (2019) Current applications and future directions of deep learning in musculoskeletal radiology. Skeletal Radiol $4: 1-5$

14. Litjens G, SánchezTimofeeva CIN, Hermsen M, Nagtegaal I, Kovacs I, van de Kaa CH, Bult P, van Ginneken B, van der Laak J (2016) Deep learning as a tool for increased accuracy and efficiency of histopathological diagnosis. Sci Rep 6:26286

15. Ke Q, Zhang J, Wei W, Połap D, WoźniakKośmider ML, Damašev̌cius R (2019) A neuro-heuristic approach for recognition of lung diseases from X-ray images. Expert Syst Appl 126:218-232

16. Chandra TB, Verma K, Singh BK, Jain D, Netam SS (2020) Automatic detection of tuberculosis related abnormalities in Chest $\mathrm{X}$-ray images using hierarchical feature extraction scheme. Expert Syst Appl 158(5):113514

17. Gupta N, Gupta D, Khanna A, Rebouças PP, Filho VH, de Albuquerque C (2019) Evolutionary algorithms for automatic lung disease detection. Measurement 140:590-608

18. Bermejo-Peláez D, Ash SY, Washko GR, Estépar RSJ, LedesmaCarbayo MJ (2020) Classification of interstitial lung abnormality patterns with an ensemble of deep convolutional neural networks. Sci Rep 10:338

19. Xiaowei X, Xiangao J, Chunlian M, Peng D, Xukun L, Shuangzhi L, Liang Y, Yanfei C, Junwei S, Guanjing L, Yongtao L, Hong Z, Kaijin X, Lingxiang R, Wei W (2020) Deep learning system to screen coronavirus disease 2019 pneumonia. arXiv:2002.09334

20. Xinggang W, Xianbo D, Qing F, Qiang Z, Jiapei F, Hui M, Wenyu L, Chuansheng Z (2020) A weakly-supervised framework for COVID-19 classification and lesion localization from chest CT. In: IEEE Transactions on Medical Imaging

21. Shuai W, Bo K, Jinlu M, Xianjun Z, Mingming X, Jia G, Mengjiao C, Jingyi Y, Yaodong L, Xiangfei M, Bo X (2020) A deep learning algorithm using ct images to screen for corona virus disease (covid19). medRxiv

22. Shan F, Gao Y, Wang J, Shi W, Shi N, Han M, Xue Z, Shen D, Shi Y (2020) Lung infection quantification of covid-19 in ct images with deep learning. arXiv preprint arXiv:2003.04655 
23. Jun C, Lianlian W, Jun Z, Liang Z, Dexin G, Yilin Z, Shan H, Yonggui W, Xiao H, Biqing Z, Kuo Z, Huiling W, Zehua D, Youming X, Yijie Z, Xi C, Lilei Y, Honggang Y (2020) Deep learning-based model for detecting 2019 novel coronavirus pneumonia on highresolution computed tomography: a prospective study. medRxiv

24. Xingyi Y, Xuehai H, Jinyu Z, Yichen Z, Shanghang Z, Pengtao X (2020) COVID-CT-Dataset: a CT scan dataset about COVID-19. arXiv:2003.13865

25. Krizhevsky A, Sutskever I, Hinton GE (2017) ImageNet classification with deep convolutional neural networks. Commun ACM 60(6):84-90

26. Nima T, Jae YS, Suryakanth RG, Todd Hurst R, Christopher BK, Michael BG, Jianming L (2016) Convolutional neural networks for medical image analysis: full training or fine tuning? IEEE Trans Med Imaging 35(5):1299-1312

27. Deng J, Dong W, Socher R, Li LJ, Li K, Fei-Fei L (2009) Imagenet: a large-scale hierarchical image database. In: CVPR

28. Tan M, Le QV (2019) Efficientnet: rethinking model scaling for convolutional neural networks. In: arXiv preprint arXiv:1905.119 46

29. Zoph B, Vasudevan V, Shlens J, Le QV (2017) Learning transferable architectures for scalable image recognition. arXiv preprint arXiv: 1707.07012

30. Szegedy C, Vanhoucke V, Ioffe S, Shlens J, Wojna Z (2016) Rethinking the inception architecture for computer vision. In: Proceedings of the IEEE Conference on Computer Vision and Pattern Recognition. 2818-2826

31. He K, Zhang X, Ren S, Sun J (2016) Deep residual learning for image recognition. In: Proceedings of the IEEE conference on computer vision and pattern recognition. 770-778
32. Hu J, Shen L, Sun G (2017) Squeeze -and -excitation networks. arXiv:1709.01507

33. Chollet F (2016) Xception: deep learning with depthwise separable convolutions. arXiv preprint arXiv: 1610.02357

34. Huang G, Liu Z, van der Maaten L, Weinberger KQ (2017) Densely connected convolutional networks. In: IEEE conference on computer vision and pattern recognition (CVPR)

35. Saining X, Ross G, Piotr D, Zhuowen T, Kaiming H (2017) Aggregated residual transformations for deep neural networks. In: The IEEE conference on computer vision and pattern recognition (CVPR). 1492-1500

36. Szegedy C, Ioffe S, Vanhoucke V, Alemi AA (2017) Inception-v4, inception-resnet and the impact of residual connections on learning. arXiv: $1602.07261 .4278-4284$

Publisher's Note Springer Nature remains neutral with regard to jurisdictional claims in published maps and institutional affiliations. 